\title{
Carcinoma of the urachus: a case report and literature review
}

\begin{abstract}
Tumors of the urachus make up less than $1 \%$ of the bladder neoplasia and approximately 20 to $35 \%$ of bladder adenocarcinomas. At present, little is known of the pathology. Etiology and pathogenesis are still uncertain and risk factors for those tumors have not been identified.

Clinical case: A 41-year-old man came to the urology service due to gross hematuria with no clot formation, accompanied by pain in the hypogastrium. Physical examination revealed an abdomen with a slightly increased volume in the hypogastrium. A mobile, nonpainful, mildly indurated tumor dependent on the umbilicus, measuring approximately $5 \mathrm{~cm}$ and not fixed to the deep planes was palpated. Abdominopelvic tomography identified a urinary bladder with wall thickening and a heterogeneous, irregular image measuring $38 \times 27 \mathrm{~mm}$ at the midline, with punctiform peripheral calcifications. Cystoscopy showed a solid tumor in the bladder dome with a diameter of $3 \mathrm{~cm}$, brown areas, and irregular edges. Treatment was transurethral resection of the bladder, with satisfactory results.
\end{abstract}

Conclusions: Carcinoma of the urachus is a rare alteration and most information on it comes from case reports and case series. The treatment of choice is partial cystectomy and en bloc resection of the urachus, which has provided good results and progression- free survival rates from 40 to $60 \%$, depending on the type of procedure performed

Keywords: carcinoma, urachus, cystectomy, partial cystectomy, hypogastrium, abdominopelvic tomography, tumor, glycosuria, proteinuria, hemoglobin
Volume 7 Issue 4 - 2019

\section{Jorge Gerardo Sandoval Téllez, Abel A Ricardez Espinosa, Miguel J Suárez Uriarte, Itzel Reyes De la Garza, Luis A Jiménez López \\ Departament of Urology, National Medical Center Lic. Adolfo Ruiz Cortines of Mexican Social Security Institute (IMSS), Mexico}

Correspondence: Jorge Gerardo Sandoval Téllez, Departament of Urology, National Medical Center Lic. Adolfo Ruiz Cortines of Mexican Social Security Institute (IMSS),Veracruz, Mexico, Email drgerardosandoval@gmail.com

Received: July 02, 2019 | Published: August 28, 2019

\section{Introduction}

Urachal tumors make up less than $1 \%$ of bladder neoplasms and approximately 20 to $35 \%$ of bladder adenocarcinomas. ${ }^{1}$ The uraco is an embryological remnant of the allantois, identified as a structure or cord fibrous from the umbilicus to the bladder dome, that is usually obstructed after birth. ${ }^{2}$ To date, little is known about carcinoma: etiology and pathogenesis are still uncertain and do not have identified risk factors. ${ }^{3}$ The treatment of choice consists of partial cystectomy, with complete exeresis of the tumor. ${ }^{4,5}$

\section{Clinical case}

A 41-year-old male patient, who went to the Urology service of the Medical Center Lic. Adolfo Ruiz Cortines (IMSS) by macroscopic hematuria, no clots formation, associated with pain in the hypogastrium. He reported weight loss of $10 \mathrm{~kg}$ in three months. Physical examination was observed the abdomen with discrete volume increase in the hypogastrium and palpation a tumor of Approximately $5 \mathrm{~cm}$, depending on the scar umbilical, mobile, not painful or fixed in the deep planes, slightly indurated. Laboratory studies reported cytometry hematics within the normal parameters; blood chemistry with glycemia of $164 \mathrm{mg} / \mathrm{dL}$, urea of $40 \mathrm{mg} / \mathrm{dL}$ and creatinine 1.1 $\mathrm{mg} / \mathrm{dL}$. The general examination of urine with glycosuria, proteinuria, hemoglobin $(+)$ and numerous erythrocytes. The ultrasound abdominal bladder showed a lobed image, of heterogeneous content adjacent to the upper wall. Abdominal-pelvic tomography (Figure 1) and (Figure 2) reported: free retroperitoneum lymphadenopathy, morphology of large vessels with normal diameter and path; in the cavity pelvic urinary bladder was observed with thickening from the wall and in the middle line the wall anterior with irregular, heterogeneous image, which was enhanced with the contrast medium, with towards the abdominal cavity and wall, $38 \times 27 \mathrm{~mm}$, with multiple calcifications Punctiform peripherals. (Figure 3 ) evidenced a tumor in the bladder dome of solid aspect, with zones of brown color, approximate diameter of $3 \mathrm{~cm}$ and irregular edges. The treatment consisted of Transurethral resection of the bladder, with results satisfactory.

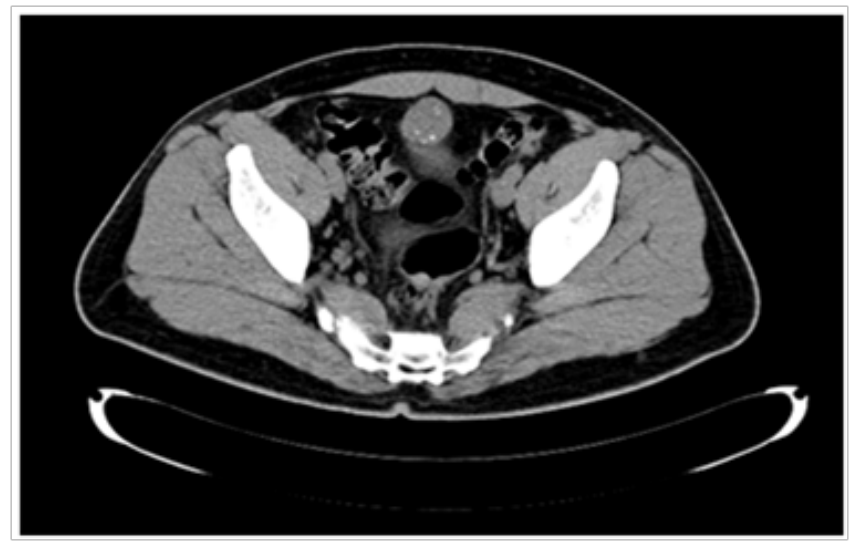

Figure I Tomography (axial) showing multiple punctate peripheral calcifications. 


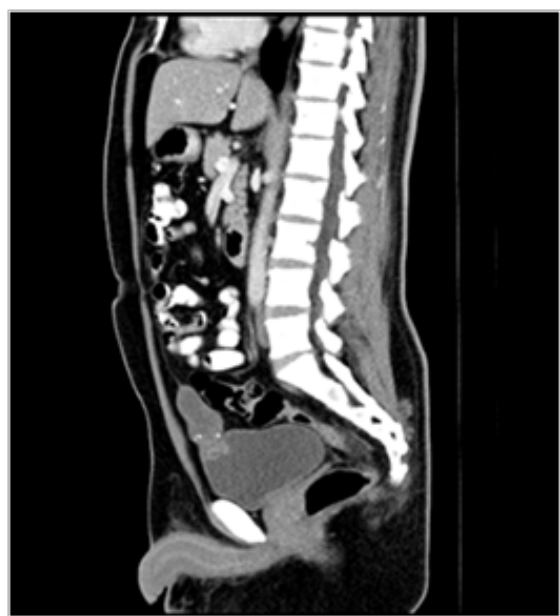

Figure 2 Contrast Tomography that evidence the tumor, with infiltration in the bladder dome.

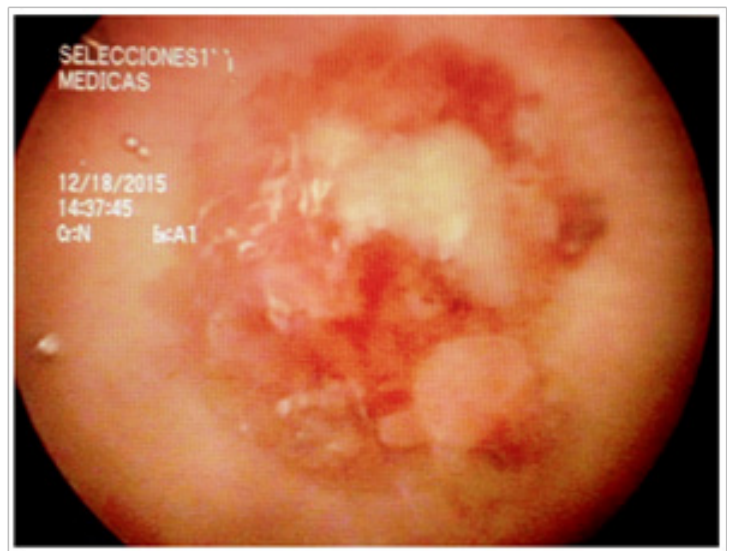

Figure 3 Bladder tumor.

\section{Histopathological findings}

The evaluated tissue was processed with the technique conventional histological (sections were made 3 microns to stain with hematoxylin and eosin). A neoplasm with groups was identified of glandular lights, mucosecretor epithelium with nuclei polarized at the base, clear cytoplasm, coexistence of mucin and areas with epithelium of Transitional type, which infiltrated adipose tissue adjacent, associated with desmoplastic reaction (inflammation and peritumoral fibrosis) (Figure 4). The sample was sent to an antibody label of cytokeratin 7 , which was positive-membranous $(+++)$, cytokeratin $20(+++)$, CDX2 positive-nuclear $(+++)$ and prostate antigen specific negative. This immunophenotype is expressed in glandular tumors of urachal origin, with glandular growth pattern, so the positivity of cytokeratin 7 rules out the gastrointestinal metastasis and the negativity for the prostate antigen rules out the possibility of metastasis of prostatic origin. Open surgical treatment was performed, accessing by the middle line, where it was found peritoneal carcinomatosis. Cystectomy was performed partial and en bloc resection of the urachus, besides lymphadenectomy and omentectomy.

The histopathology report was: adenocarcinoma mucoproducer with scarce tubular glands, moderately differentiated, which affected the mucosa and the wall to the muscular layer of the bladder, in addition to two tumor implants in the omentum of 3 and $5 \mathrm{~cm}$. The lymph nodes showed, only, sinusoidal hyperplasia reactive. The patient received adjuvant chemotherapy with cisplatin and gemcitabine. After 10 months of treatment the disease persisted, with ganglionar activity on the left side of the vneck, ipsilateral armpit, just like in the area para-aortic and hepatic, so the scheme to paclitaxel and carboplatin. Adverse effects caused by chemotherapy they were little tolerated by the patient; by so, he decided to suspend the treatment after one month after starting it. At present receives chemotherapy with capecitabine for palliatives and tramadol-paracetamol to control the pain.

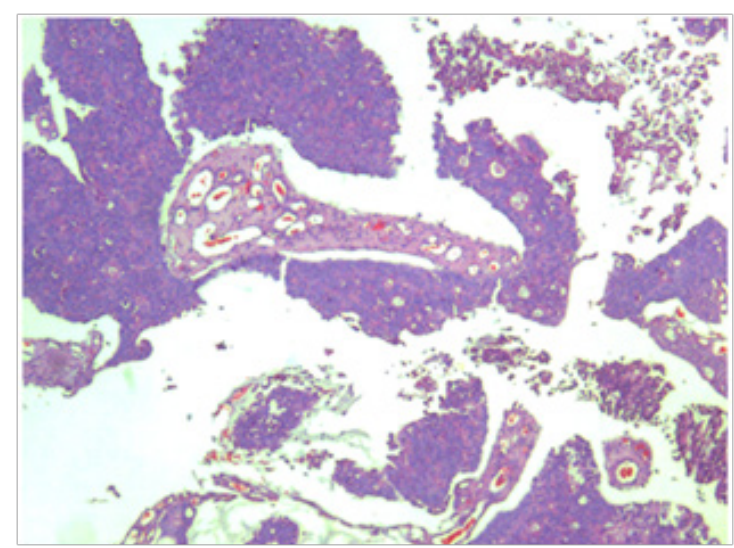

Figure 4 Cytology showing groups of glandular lights, mucosecretor epithelium with nuclei polarized at the base and clear citoplasm.

\section{Discussion}

Urachal carcinoma is an exceptional tumor, first described in 1931 by Begg. ${ }^{1}$ It comprises $0.17-0.34 \%$ of the neoplasms of bladder and its annual incidence is estimated at 1 every 5 million patients. ${ }^{4}$ It affects with greater frequency to men (1.8:1) between 50 and 60 years of age. $^{5}$ To date, there have been no identified risk factors to suffer from this type tumors, likewise has been ruled out that the persistence of the permeability of the uraco represent a predisposing factor for the expression of the carcinoma. There are less than 400 cases reported in the bibliography from its first description (1931). ${ }^{5}$ Due to its low incidence it is not a disease included in the practice guidelines American or European clinics, so that the diagnosis and treatment are unclear. Adenocarcinomas constitute the histological type most frequent of urachal tumors $(90 \%)$ and of these, $63.6 \%$ are producers of mucin, $4.5 \%$ contain transitional cells and $2.2 \%$ mixed microcytic pattern, of transitional cells and adenocarcinoma. The remaining $10 \%$ of urachal tumors are urothelial (4\%), sarcomatous $(4 \%)$ and undifferentiated $(2 \%)$. Clinical manifestations go unnoticed and are usually derived from the invasion of the tumor at neighboring structures; therefore, it is often they diagnose in advanced stage. Hematuria (microscopic or macroscopic) is the symptom predominant, even in $85 \%$ of cases. Pain is also usually felt in the region periumbilical $(8.5 \%)$, foul or bloody discharge by the navel and mucous secretion by the urethra $(17 \%)$; in some cases it can be felt the tumor $(17 \%))^{7}$

The initial diagnosis is established with studies of imaging, such as computed tomography, magnetic resonance and ultrasound. The find most frequent tomographic is the identification of intravesical calcifications $(70 \%)$. cystoscopy a tumor located in the bladder dome or in the anterior wall $(91.5 \%)$; macroscopically it has a polypoid aspect or ulcerated Urinary cytology is positive in $38 \%$ of cases. ${ }^{7}$ Some studies have reported high concentration of the 
carcinoembryonic antigen, $\mathrm{Ca} 19-9$ and $\mathrm{Ca}-125$, mainly when the origin of these tumors is enteric; but nevertheless, are not useful to establish the diagnosis or follow-up. ${ }^{9}$ Immunohistochemistry expresses markers for cytokeratin 20 (CK20) and the CDX-2 gene in $100 \%$ of uraco tumors and cytokeratin 7 up to $50 \%{ }^{10}$ The treatment of choice consists of cystectomy partial with complete resection of the urachus and the belly button in block. Survival of patients with partial cystectomy is similar to that of subjects with radical surgery. The procedure surgical should focus on the preservation of the bladder. Survival at five years varies from 40 to $60 \% .{ }^{5}$ Factors of poor prognosis include: coexistence of adenopathies, distant metastasis, positive surgical margins, tumor size, Differentiation of cells of the seal ring, mucin production, peritoneal involvement, Umbilectomy, lymphadenectomy and cystectomy partial versus radical (the latter do not show significant differences in terms of survival respect). ${ }^{2}$

Coadjuvant treatment with chemotherapy It is essential in all cases with positive regional ganglia. ${ }^{11}$ The protocols of chemotherapy combined with cisplatin (methotrexate, vinblastine, doxorubicin and cisplatin or gemcitabine-cisplatin) represent the first line in patients with carcinoma urothelial of bladder and urachus. The carcinoma of the uraco shares histological and clinical similarity with colon adenocarcinoma; so, Chemotherapy with 5-fluorouracil also It can be indicated in patients with this neoplasm. Promising results have been published with the combination of 5-fluorouracil, leucovorin, gemcitabine and cisplatin. ${ }^{2}$ To date, there is no scientific evidence to support the indication of radiotherapy or chemotherapy as the best treatment option adjuvant. ${ }^{7}$ Studies that evaluate the adjuvant chemotherapy have included few patients, established therapeutic schemes variables and have not contributed any level of evidence. ${ }^{3}$

\section{Conclusion}

Carcinoma of the urachus is a rare alteration and most information on it comes from case reports and case series. The treatment of choice is partial cystectomy and en bloc resection of the urachus, which has provided good results and progression- free survival rates from 40 to $60 \%$, depending on the type of procedure performed. Factors of poor prognosis include distant metastasis and coexistence of ganglia lymphatics there is little evidence related to the chemotherapy schemes and their repercussion in global survival.

\section{Acknowledgment}

None.

\section{Conflicts of interest}

The author declares there is no conflict of interest.

\section{References}

1. Begg RC. The urachus: Its anatomy, histology and development. J Anat. 1930;64(Pt 2):170-183.

2. Szarvas T, Módos O, Niedworok C, et al. Clinical, prognostic, and therapeutic aspects of urachal carcinoma-A comprehensive review with meta-analysis of 1,010 cases. Urol Oncol. 2016;34(9):388-398.

3. Cruz P, Ramírez M, Iborra I, et al. Adenocarci- noma de uraco: ¿qué tanto sabemos? Rev Mex Uro. 2014;74(5):301-307.

4. Sheldon CA, Clayman RV, González R, et al. Malignant urachal lesions. J Urol. 1984;131(1):1-8.

5. Herr HW, Bochner BH, Sharp D, et al. Urachal carcinoma: contemporary surgical outcomes. J Urol. 2007;178(1):74-78.

6. Molina JR, Quevedo JF, Furth AF, et al. Predictors of survival from urachal cancer. A Mayo Clinic study of 49 cases. Cancer. 2007;110(11):2434-2440.

7. Ashley RA, Inman BA, Sebo TJ, et al. Urachal carcinoma: Clinicopathologic features and long term outcomes of an aggressive malignancy. Cancer. 2006;107(4):712-720.

8. Thali-Schwab CM, Woodward PJ, Wagner BJ. Computed tomographic appearance of urachal adenocarcinomas: Review of 25 cases. Eur Radiol. 2005;15(1):79-84.

9. Siefker-Radtke AO, Gee J, Shen Y, et al. Multimodality management of urachal carcinoma: The M.D. Anderson experience. $J$ Urol. 2003;169(4):1295-1298.

10. Paner GP, McKenney JK, Barkan GA, et al. Immunohisto- chemical analysis in a morphologic spectrum of urachal epithelial neoplasms: Diagnostic implications and pitfalls. Am J Surg Patho. 2011;35(6):787798.

11. Meeks JJ, Herr HW, Bernstein M, et al. Preoperative accuracy of diagnostic evaluation of the urachal mass. J Urol. 2013;189(4):1260 1262 . 